\title{
Design of Easily Removable Lattice-Based Support Structures for L-Pbf
}

1, Omid Zarei , Ole Geisen , 2 Gaurav Jitendra Pawar , 2 Stephan Ziegler ,1-4 Johannes Henrich Schleifenbaum 1, 4

\begin{abstract}
Laser powder bed fusion (L-PBF) is a type of additive manufacturing technology that processes metal powders into a component. Support structures are an essential part of the LPBF process as they transfer the laser-induced heat during and shortly after the process to the substrate, sustaining positional accuracy of downward facing surfaces of the component. Since the use of support structures is inevitable, optimized designs for them are crucial in realizing more sustainable production process. In a serial production setup, reducing the lead time and cost of a non-value-added process step like support structure removal is of significance when improving the overall business case and competitiveness.

The goal of this study was to verify the applicability of lattice-based support structures for L-PBF. To achieve this, different lattice types as support structures were designed. They were tested, compared and verified for a Siemens gas turbine component. The results showed that the generated lattice-based support structures could be suitable for L-PBF. The supports had to be designed appropriately such that they could preserve the geometry of the part. Furthermore, they had to have a short fabrication time and to be removable easily, preferably without machining or sawing.
\end{abstract}

\section{Keywords}

Additive manufacturing, laser powder bed fusion, support structures, lattice structures, easily removable, overhang

\section{Introduction}

Laser powder bed fusion (L-PBF) is an additive manufacturing technology based on layer-by-layer material additions of melted metal powder in an inert gas environment [1]. One important characteristic of this technology is its capability to produce components with highly complex geometries [2]. The L-PBF process requires support structures which connect a component to the substrate plate. These support structures have two main functions: The first is to dissipate the laser-induced heat while melting the material; the second is to constrain the solidified material to ensure geometrical accuracy of the component [3]. The latter is especially relevant for the downward facing surfaces with low overhang angles which might collapse without support structures. Overhang angle is defined as the angle between a tangent plane to a downward facing surface (of a component) and the substrate plate; the threshold overhang angle, describing the manufacturability of a feature without support structures, is defined for a specific L-PBF process and material as the minimum overhang angle which is

\footnotetext{
${ }^{1}$ Chair for Digital Additive Production, RWTH Aachen University, Steinbachstrabe 15, 52074 Aachen, Germany

${ }^{2}$ Siemens AG, Power and Gas Division, Huttenstrabe 12, 10553 Berlin, Germany

${ }^{3}$ RWTH Aachen University, Templergraben 55, 52062 Aachen, Germany

${ }^{4}$ Fraunhofer Institute for Laser Technology, Steinbachstrabe 15, 52074 Aachen, Germany
} 
manufacturable without sacrificial support [4]. A considerable amount of support structures may be necessary for a component to ensure its manufacturability. They need to be removed after the process, which adds significant time and cost to the L-PBF production [5]. This motivates to design support structures with low volume fraction in an easily removable manner, preferably detachable without machining or sawing. One possible solution is to use lattice-based support structures. Lattices as support structures are effective in terms of volume reduction leading to cost savings and lower build time. They can accomplish both the purposes of support structures, namely heat dissipation as well as constraining the solidified material for the sake of geometric accuracy during the L-PBF process. Hussein et al. [5] studied support structures with Schoen gyroid and Schwartz diamond lattice types for a cantilever component made of titanium alloy Ti6Al4V. They obtained guidelines for choosing volume fraction and cell size of the studied lattice types to design more efficient support structures. Jarvinen et al. [3] reported that support structures strongly affect manufacturability and the final quality of the component. They studied the removability of web and tube supports and found that the web type can be detached easily. Furthermore, Gan and Wong [6] investigated pin, $\mathrm{Y}$ or inverted $\mathrm{Y}$ support structures for the L-PBF process. They concluded that the inverted $\mathrm{Y}$ support structures outperforms the other two types in terms of minimum overhang support areas and maximum space between the (pin, Y or inverted Y) units. It is often easier and less costly to remove lattice-based support structures than other kinds of support structures like block-based types [7]. This might be related to the nature of lattice supports, providing a number of small discrete bonds with both the part and the substrate plate through its struts. Moreover, lattice-based support structures show good manufacturability characteristics [8]. However, there is still room for improvement in the proper design of lattice-based support structures to be widely used in L-PBF [8].

This paper investigates the design and verification of lattice-based support structures for the L-PBF process. To substitute the conventional production method with the L-PBF process for the resonator box of a Siemens gas turbine, lattice support structures are designed to be effortlessly removable. The chosen component is a hollow structure; therefore, it must be de-powdered after fabrication. If the support structure would be easily removable such that it can be detached with a chisel and preferably without machining or sawing, the depowdering could be carried out conveniently by manual shaking. A few variant forms of lattice structures are introduced and used for the design of support structures. They will be compared in terms of their manufacturability, removability and fabrication time and the most promising type(s) will be determined. The results of this work can serve as a practical guideline for using lattice-based support structures in the L-PBF process.

\section{Methodology}

The methodology used to design and manufacture the lattice support structures is explained in the following sections. 


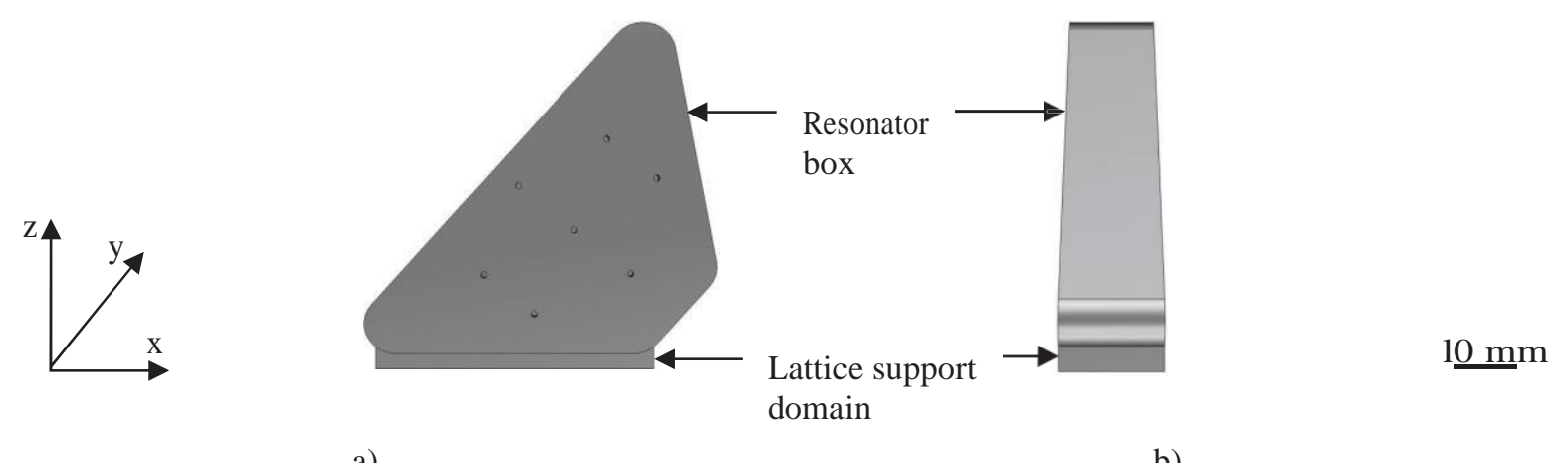

a)

b)

Fig. 1 Domain of lattice support structures for the resonator box: a) Front view, b) Left view

\subsection{Design of lattice support structures}

A gas turbine resonator box was used as the application case. The resonator box model was constructed using Siemens NX 12. The model was exported as an STL file with chordal and angular tolerance values of $0.0025 \mathrm{~mm}$ and $1.0^{\circ}$, respectively. It was then imported in Materialise Magics software to prepare the build job. The box was placed $3 \mathrm{~mm}$ above the substrate plate, and it was supported with lattice structures. The threshold overhang angle of $40^{\circ}$ was used to determine the required area for the supports. The resonator box with the domain of lattice-based support structures is shown in Fig. 1. The building orientation was already decided as demonstrated in Fig. 1.

For the support domain of the box, five different lattice structures were designed. Lattice structures were generated with the lattice generator tool developed at the Chair for Digital Additive Production, RWTH Aachen University. The unit cells of lattices are illustrated in Fig. 2 with strut diameters of $1 \mathrm{~mm}$. The cells are cuboid with the height of $3 \mathrm{~mm}$ and the other two dimensions were chosen close to $3 \mathrm{~mm}$, within the interval of 2.95 to $3.30 \mathrm{~mm}$, to make the lattice supports include struts near the edges and corners of the supported faces. In general, this is not achievable using cubic unit cells. Those dimensions were kept constant for all the lattices. Preliminary experiments showed that a strut diameter smaller than $0.5 \mathrm{~mm}$ could fail to dissipate the generated heat during the L-PBF manufacturing of the resonator box. On account of this, the strut diameter was set to either 0.7 or $1 \mathrm{~mm}$. The strut diameters were identical for each lattice structure.

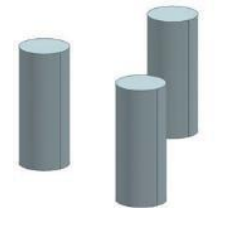

a)

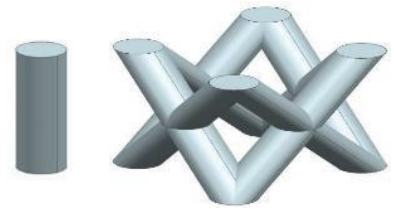

b)

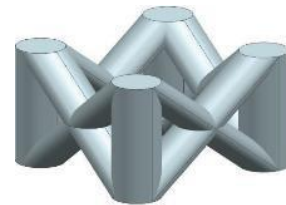

c)

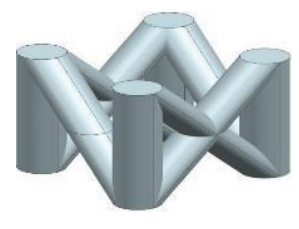

d)

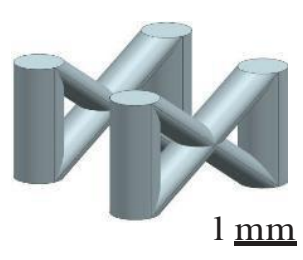

e)

Fig. 2 Lattice types: a) Bar, b) Lozenge, c) Mixed, d) One-Sided, and e) Planar

The experiment was prepared for the resonator box with the designed lattice supports. Each type of lattice support was used twice in the same build job to investigate the reproducibility of the structures. The experimental setup is shown in Fig. 3. In the figure, volume fraction is defined as the volume occupied by the lattice structures divided by the volume of the lattice domain. Lattice domain is the volumetric space including lattice struts 
and non-filled spaces trapped between the struts all together. Note that the volume fractions were determined based on the design parameters.

\subsection{Manufacturing process}

The L-PBF system EOS M 290 was used to carry out the experiments for this study. It has the build volume of $250 \times 250 \times 300 \mathrm{~mm}^{3}$. The machine uses an ytterbium fiber laser with a peak power output of $400 \mathrm{~W}$ and a maximum laser scanning speed of $7000 \mathrm{~mm} / \mathrm{s}$. Argon gas is circulated in the building chamber of the machine to create an inert environment during the operation. The machine also deploys a flexible recoater lip to distribute the metal powder uniformly on the substrate plate.

Table 1 Chemical composition of IN718 in weight percent [9]

\begin{tabular}{|c|c|c|c|c|c|c|}
\hline $\mathbf{N i}$ & $\mathbf{C r}$ & $\mathbf{N b}$ & $\mathbf{M o}$ & $\mathbf{T i}$ & $\mathbf{A l}$ & $\mathbf{C o}$ \\
\hline $50.0-55.0$ & $17.0-21.0$ & $4.75-5.5$ & $2.8-3.3$ & $0.65-1.15$ & $0.2-0.8$ & $:: 1.0$ \\
\hline $\mathbf{C u}$ & $\mathbf{C}$ & $\mathbf{S i}, \mathbf{M n}$ & $\mathbf{P}, \mathbf{S}$ & $\mathbf{B}$ & $\mathbf{F e}$ & $\mathbf{p}\left(\mathbf{g} / \mathbf{c m}^{\mathbf{3}}\right)$ \\
\hline$:: 0.3$ & $:: 0.08$ & each $:: 0.35$ & each $:: 0.015$ & $:: 0.006$ & balance & $\min .8 .15$ \\
\hline
\end{tabular}

The powder material used for this study was Inconel 718 (IN718). The chemical composition of the IN718 is given in Table 1 . The powder particle size was in the range of 20 - $63 \mu \mathrm{m}$. The layer thickness of $60 \mu \mathrm{m}$ was used for the build job. The dominant thickness of support structures, distance between the substrate plate and the horizontal downfacing surface, was deliberately chosen as divisible by the layer thickness. Therefore, the interface between the part and the support structure would be the interface between two successive layers as well.

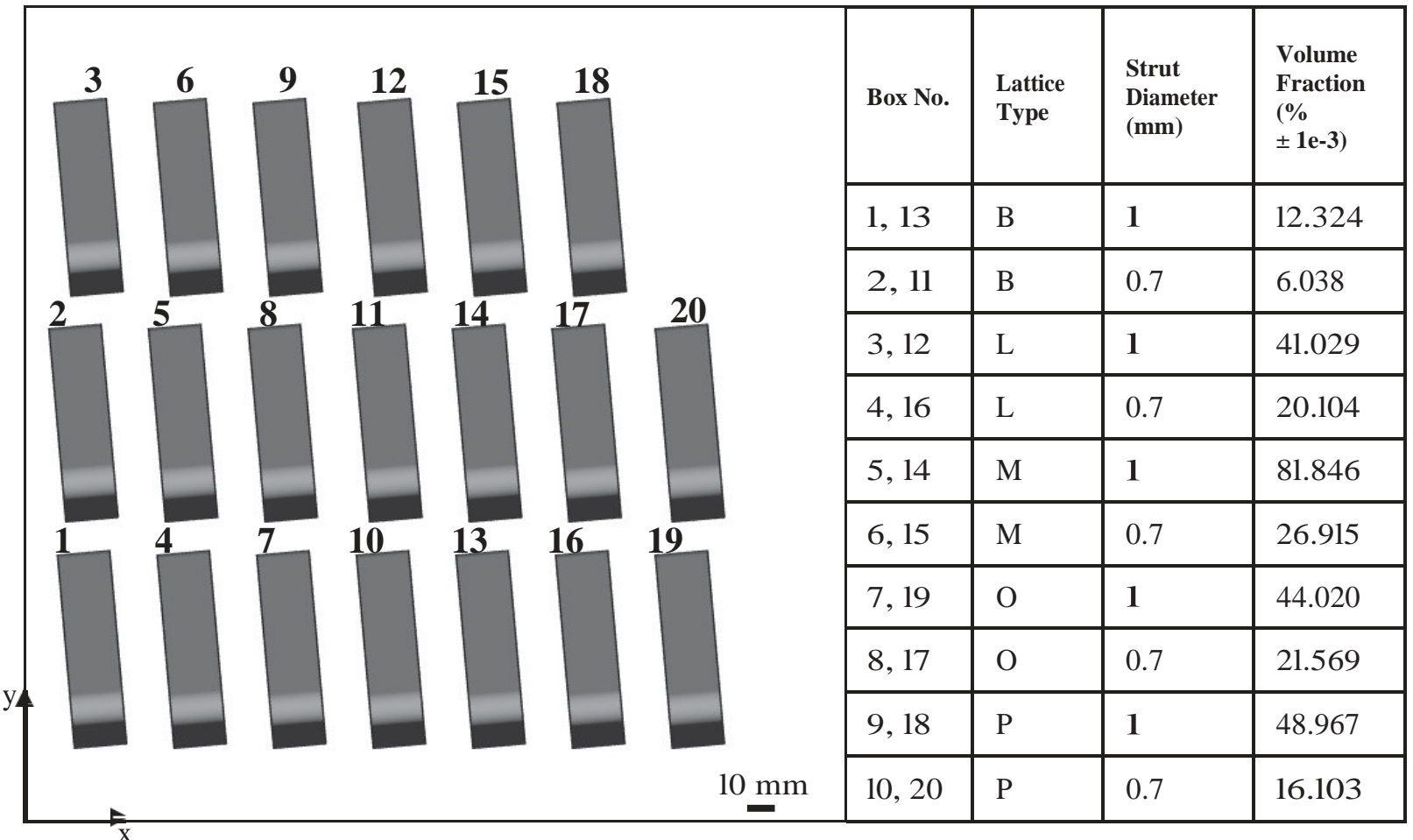

Fig. 3 Build job setup for the experiment (B: Bar, L: Lozenge, M: Mixed, P: Planar, O: OneSided) 
In the experiment, the boxes were built without down-skin process parameters. The hatch and contour process parameters of the resonator box are characterized to satisfy the requirement of minimum material density of $99.5 \%$. With the exception of laser power, the support process parameters are chosen to be the same as that of the resonator box. The laser power for the lattice supports is chosen as $80 \%$ of that for the resonator box. The described procedure can be used to determine the process parameters for the fabrication of lattice structures, especially for sacrificial support lattices since their quality is of less concern. The lattice supports were fabricated without the contour and up-/down-skin process parameters. This results in lower production time.

\section{Results and discussion}

The boxes were built without down-skin parameters while supported by lattice structures as shown in Fig. 4. Different lattice types were compared in terms of three different criteria. They include removability, geometric preservation and fabrication time. Table 2 summarizes the behavior of the lattices with respect to the three aforementioned criteria of lattice supports.

The visual inspection was used for damage analysis and geometric preservation. Out of all the lattice structures manufactured, only four cases namely lozenge, mixed and planar types with a strut diameter of $1 \mathrm{~mm}$ and the mixed type with a strut diameter of $0.7 \mathrm{~mm}$ showed acceptable geometric accuracy with almost no visible support-related defects, especially not in the corners as marked with rectangles in Fig. 5. The other samples had defects on the rounded surfaces of both corners. This can be related to the insufficient supports in those areas. Fig. 6 shows that when the homogeneous lattice is used, the distance between the corner struts, denoted by ' $b$ ', is greater than that at the downfacing horizontal surface, denoted by 'a'. This might explain why the near-corner areas are more prone to defects than the horizontal surface. The struts in the corner should transfer more heat than the struts at the horizontal surface since they are supporting bigger local surface (w: width of the box; local area in the corner $=\mathrm{w} \mathrm{x} \mathrm{b}$ ] local area at the horizontal surface $=\mathrm{w} x$ a). This implied that the heat-induced stresses were larger in the corners than at the horizontal surface; consequently, more deformation and/or defects in the corners were created.

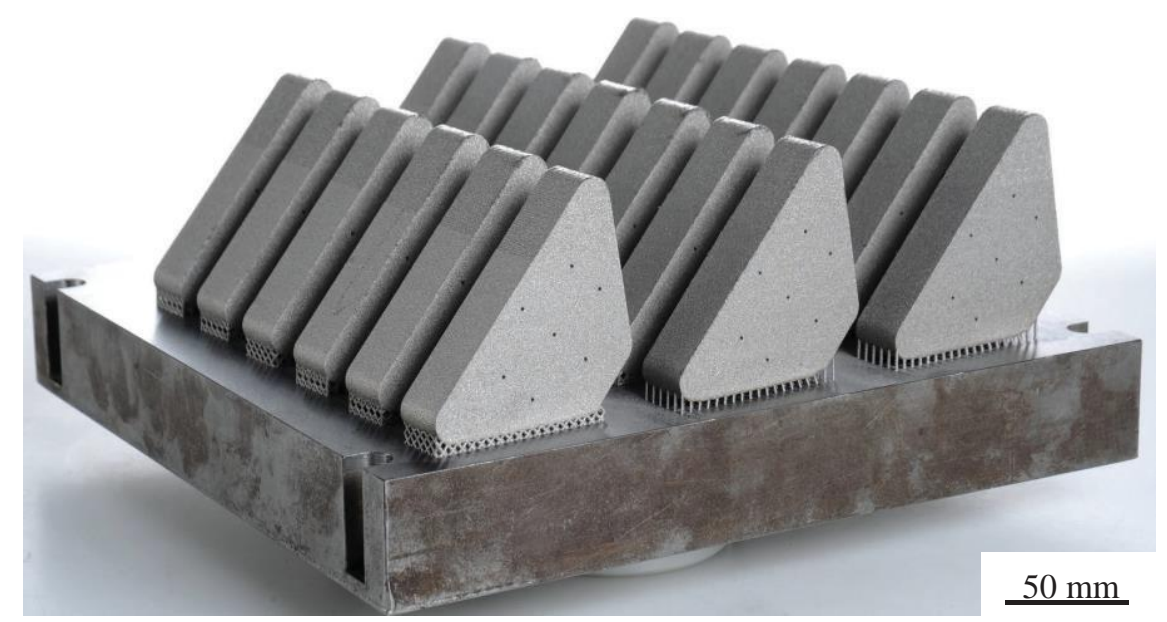

Fig. 4 The L-PBF fabrication of the resonator boxes

Both bar lattice supports with strut diameters of 0.7 and $1 \mathrm{~mm}$ showed rather large deflections of the struts, as shown in Fig. 7. This was greater for the bar support with a strut 
diameter of $0.7 \mathrm{~mm}$. The deflection of supports during the L-PBF process adversely affect the geometric accuracy of a part. Therefore, the strut diameter of bar supports should be chosen large enough and/or the unit cell dimensions of this support type should be chosen small enough such that the deformation of the supports during the process is kept low.

Table 2 Comparison of lattice supports

\begin{tabular}{|l|l|l|l|l|}
\hline $\begin{array}{l}\text { Lattice } \\
\text { Type }\end{array}$ & Strut Diameter & $\begin{array}{l}\text { Geometric Feature } \\
\text { Preservation }\end{array}$ & Removability & $\begin{array}{l}\text { Fabrication time } \\
(\mathbf{s} \pm \text { 1e-3) }\end{array}$ \\
\hline Bar & $1 \mathrm{~mm}$ & No & Easy & 120.547 \\
\hline Bar & $0.7 \mathrm{~mm}$ & No & Easy & 74.838 \\
\hline Lozenge & $1 \mathrm{~mm}$ & Yes & Difficult & 263.684 \\
\hline Lozenge & $0.7 \mathrm{~mm}$ & No & Medium & 178.796 \\
\hline Mixed & $1 \mathrm{~mm}$ & Yes & Difficult & 316.654 \\
\hline Mixed & $0.7 \mathrm{~mm}$ & Yes & Difficult & 221.392 \\
\hline One-Sided & $1 \mathrm{~mm}$ & No & Difficult & 277.551 \\
\hline One-Sided & $0.7 \mathrm{~mm}$ & No & Easy & 189.968 \\
\hline Planar & $1 \mathrm{~mm}$ & Yes & Difficult & 222.355 \\
\hline Planar & $0.7 \mathrm{~mm}$ & No & Easy & 150.128 \\
\hline
\end{tabular}

The ease of removability is graded as easy, medium and difficult based on the force required to remove the boxes with a chisel or the necessity of removal with sawing or machining. To label removability of the boxes, they were removed by a single person who graded them qualitatively. Easy describes the removability with the chisel and hammer; medium is interpreted as the removable similar to the easy case but with more effort; difficult implies that the supports cannot be removed with the chisel and hammer and they need to be sawed or machined. All the lattice supports with strut diameters of $1 \mathrm{~mm}$ were hardly removable with the chisel and hammer because of their strong connection with the resonator box; and hence, had to be removed using sawing or machining. Bar supports were easy to be removed because of the structure's simplicity and greater freedom in chisel placement. The other results are listed in Table 2. One important factor in the ease of removability is related to the topology of lattice structures. More complex structures and small cell sizes provide less freedom for chisel placement at appropriate orientations; and thus, indicate more difficult removability.

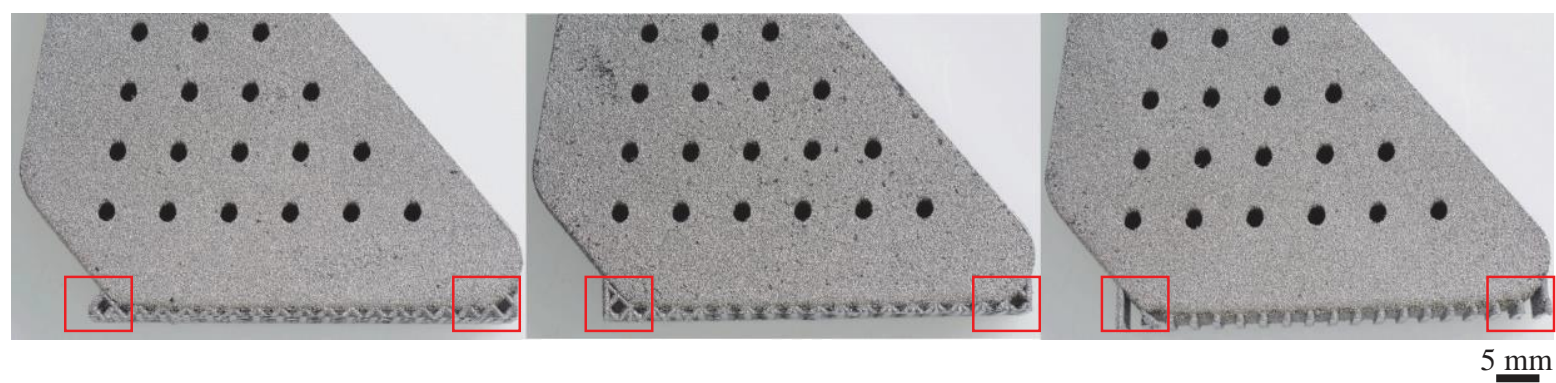

a)

b)

c)

Fig. 5 Geometricfeature preservation with strut diameters oflmm: a) Lozenge, b) Mixed, and c) Planar

The easy- or medium-removable lattice structures resulted in damaged geometric features of the boxes, as illustrated in Fig. 8 where the defective areas are marked with rectangles. The remaining portions of these lattice supports could also easily be removed 
using sand paper. Contrary to these lattices, those which were removable by sawing showed minimum defects on the boxes, as shown in Fig. 5.

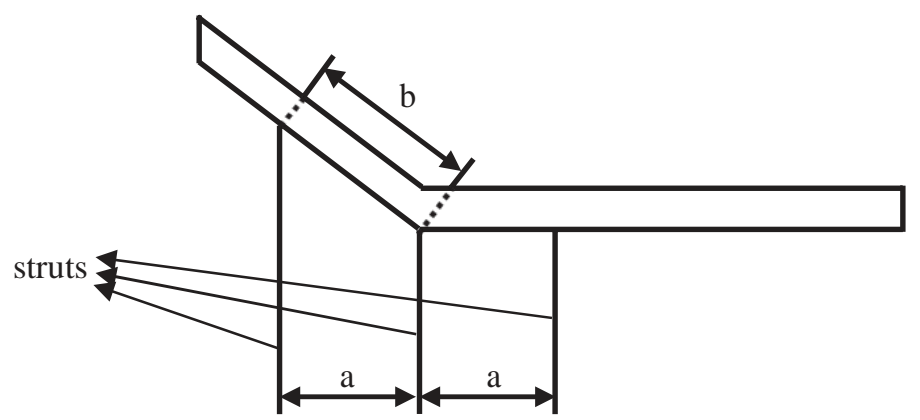

Fig. 6 Sketch of lattice support structures in the left corner

A comparison between fabrication times of the lattice supports was made in Table 2. Fabrication time is defined as the laser exposure time required to build the lattice supports including the passive time between scan vectors. It excludes the time required for the distribution of powder using a recoater in each layer and this time is constant for all the resonator boxes in a single build job. It also excludes the time required for moving the piston upward to provide the metal powder for each layer. This is also the same for all the boxes. The fabrication times were computed using the software developed at the Chair for Digital Additive Production, RWTH Aachen University. The results are listed in Table 2. They show that the fabrication times depend on the complexity of the lattice support structure and the strut diameter. The bar lattice supports were the fastest among the experimentedsupports.

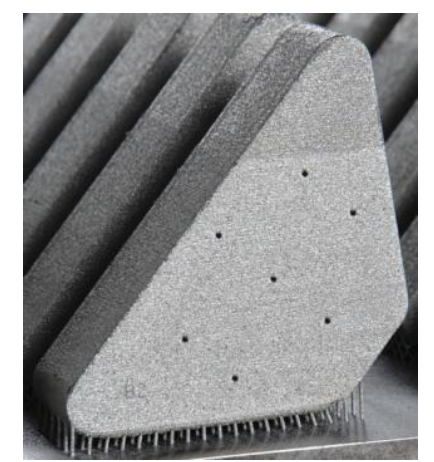

a)

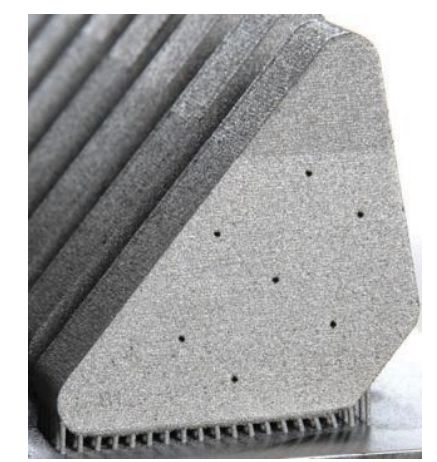

b)
$5 \underline{\mathrm{mm}}$

Fig. 7 Deflections of the bar support structures with a strut diameter of: a) $0.7 \mathrm{~mm}, \mathrm{~b}) 1 \mathrm{~mm}$

Three metrics were used for the comparison between all the generated lattice supports. The results demonstrated that the bar supports were the fastest to build and easily removable among the designed supports; however, they were unfit to preserve geometric features. They are also prone to deflection and require a relatively high design effort. More complicated structures, like the mixed type, preserved geometric features even with the smaller diameter size, but they required longer fabrication time and were difficult to remove.

\section{Conclusions and outlook}

In this study, five types of lattice structures with two different strut diameters of 0.7 and $1 \mathrm{~mm}$ were designed as support structures. The used lattices were homogeneous throughout the support domain, signifying the same lattice type with the same parameters across the domain. They were fabricated through the L-PBF process in which lattice supports were 
produced using only hatch scanning vectors for the sake of fast processing time. They were compared in terms of three criteria: geometric feature preservation, ease of removability, and fabrication time.

One remarkable observation was that the diameter of the struts played an important role in the behavior of the lattice supports. While smaller lattice supports were mostly easily removable, they were unable to satisfy the required geometric accuracy. In addition to the strut diameter, the lattice type had a significant impact on the overall behavior of the supports.

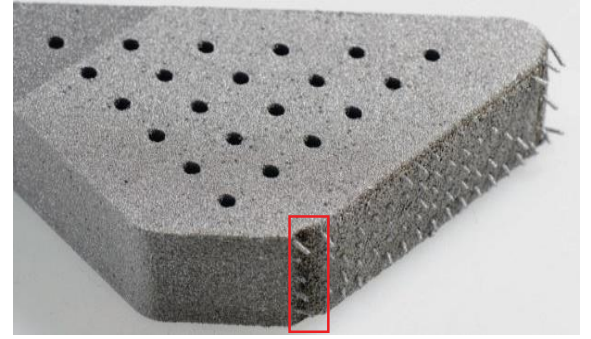

a)

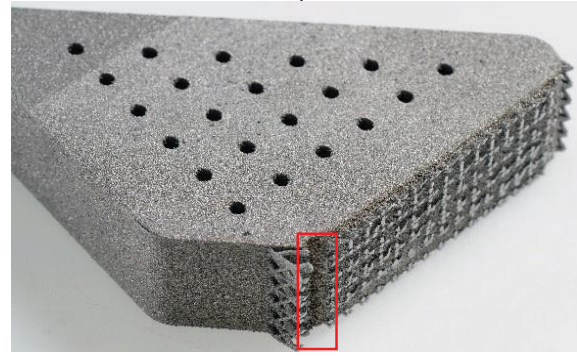

c)

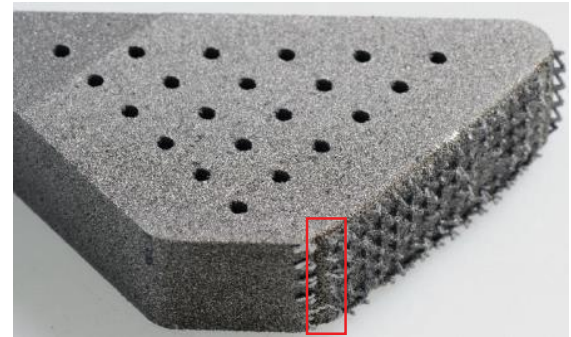

b)

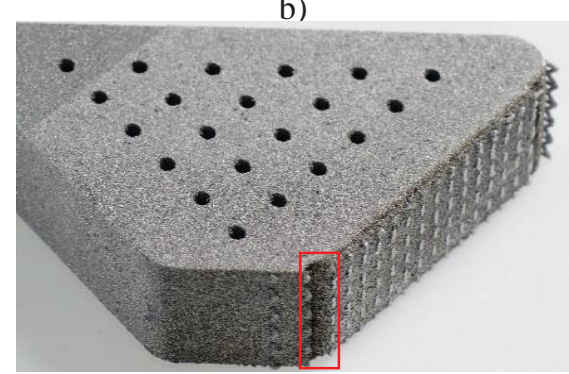

d)

Fig. 8 Easily removed lattice supports with strut diameters of $0.7 \mathrm{~mm}$ : a) Bar, b) Lozenge, c) One-Sided, and d) Planar

The results showed that among the fabricated boxes, those with the bar supports were the fastest to build and the easiest to remove; however, the geometric preservation was not satisfactory. The problems were mainly in the corners of the boxes and can be related to the insufficient support in these areas. Another issue was the deflection of the bar supports which can negatively affect the global geometric accuracy.

Mixed lattice supports showed highly acceptable geometric preservation while the removability and fabrication time of these structures were difficult and long, respectively, compared to the other designed lattice supports. More complex structures may allow smaller strut diameter, such as the mixed type, while preserving the geometric accuracy.

The geometric preservation is of great importance for the application in hand; acceptable accuracy was provided by only four lattice supports among which the mixed support with a strut diameter of $0.7 \mathrm{~mm}$ was chosen as the best variant. It had the smallest volume fraction of $26.915 \%$ and the shortest fabrication time among the acceptable variants. The other three were the lozenge, one-sided, and mixed supports with strut diameters of $1 \mathrm{~mm}$. Their fabrication time can be sorted in ascending order as planar, lozenge and mixed supports. The mixed type with a strut diameter of $1 \mathrm{~mm}$ was the worst acceptable option since not only its fabrication time was long, but also the volume fraction was extremely high.

Interestingly, it was observed that the homogeneous lattice supports might not be the efficient lattice supports since the required material amount of a part over a supporting cell can vary based on the surface orientation and/or local thickness of the part. This motivates the 
use of either different lattice types or to utilize the adaptive strut diameter in different local supporting areas. This needs to be further studied.

To conclude, it can be stated that the L-PBF production of resonator boxes can be improved using tailored lattice supports instead of solid support structures. A significant reduction both in lead time and in cost was achieved by designing lattice support structures according to the component and material requirements - with additional potential for adapting the lattice geometries such that they efficiently support the part during the L-PBF process.

\section{References}

[1] Gibson, I., Rosen, D. W., and Stucker, B., Additive Manufacturing Technologies: 3D Printing, Rapid Prototyping, and Direct Digital manufacturing, 2nd., Springer, New York, (2015), pp. 1-498.

[2] F. T. Piller, R. Poprawe, H. J. Schleifenbaum, G. Schuh, S. Barg, C. Dolle, C. Hinke, M.). Jank, R. Jiang, W. Meiners, M. Riesener, J. Schrage, S. Ziegler, Introducing a Holistic Profitability Model for Additive Manufacturing: An Analysis of Laser-powder Bed Fusion, IEEE International Conference on Industrial Engineering and Engineering Management (IEEM), Bangkok, (2018), p. 1730

[3] J.-P. Jarvinen, V. Matilainen, X. Li, H. Piili, A. Salminen, I. Makela, O. Nyrhila, Characterization of Effect of Support Structures in Laser Additive Manufacturing of Stainless Steel, Physics Procedia (2014, Volume 56), p. 72

[4] J. Jiang, J. Stringer, X. Xu, R. Y. Zhong, Investigation of printable threshold overhang angle in extrusion-based additive manufacturing for reducing support waste, International Journal of Computer Integrated Manufacturing (2018, Volume 31), p.961

[5] A. Hussein, L. Hao, C. Yan, R. Everson, P. Young, Advanced lattice support structures for metal additive manufacturing, Journal of Materials Processing Technology (2013, Volume 213), p. 1019

[6] M. X. Gan, C. H. Wong, Practical support structures for selective laser melting, Journal of Materials Processing Technology (2016, Volume 238), p. 474

[7] S. Goutianos, Selective Laser Melting of Hot Gas Turbine Components: Materials, Design and Manufacturing Aspects, IOP Conference Series: Materials Science and Engineering (2017, Volume 219), p. 012022

[8] K. Zeng, Optimization of support structures for selective laser melting, Electronic Theses and Dissertations (2015)

[9] Material data sheet: EOS NickelAlloy IN718 